\title{
The influence of alkalising amines on the film formation by oleyldiamine
}

\author{
W. Hater, ${ }^{1 *}$ P. Kraft $^{1}$ and C. Forêt ${ }^{2}$ \\ ${ }^{1}$ Kurita Europe APW GmbH, Giulinistraße 2, 67065 Ludwigshafen, Germany \\ ${ }^{2}$ Kurita France SAS, Z. I. Le Roineau, 72500 Vaas, France \\ E-mail: Wolfgang.Hater@,Kurita.eu
}

\section{Abstract}

The film formation of film forming amines (FFAs), commonly used as corrosion inhibitors in water/steam cycles, has been subject of various studies. However, less is known of the impact of alkalising amines (AA) on the film formation. AAs have a solubilising effect on the FFA and as a consequence might indirectly influence the properties of the film.

Therefore, the film formation of oleyldiamine, a commonly used FFA, on carbon steel has been studied with electrochemical impedance spectroscopy (EIS) in defined test water, containing $200 \mathrm{mg} \mathrm{L}^{-1}$ of $\mathrm{NaCl}$, with and without the presence of different AAs. The studies have been carried out at two different $\mathrm{pH}$ values over an exposure time up to $5 \mathrm{~h}$.

All EIS spectra of the blank show one semi-circle, whereas two distinct semi-circles have been observed in the spectra of the test solutions with FFA. Therefore, the steel solution interface has been described by two different equivalent circuits using resistances and constant phase elements. All EIS spectra could be fitted with good agreement to the experimental data. The spectra showed significant and systematic changes throughout the exposure time.

All polarisation resistances measured with FFA at $\mathrm{pH} 9.0$ are much higher compared to the data obtained at $\mathrm{pH}$ 8.2. The polarisation resistance of the blank solution is strongly decreasing over time following roughly an exponential law and almost comes to a steady state after 5 hours. In contrast to this the solutions containing FFA show a linear increase of the polarisation resistance over time. Obviously the film formation has not yet completely been finished within the experimental period.

The FFA solutions containing the two different AA show a similar behaviour over time and almost the same high polarisation resistance, reflecting the excellent corrosion protection. Overall, the data indicate that the film obtained with oleylamine in the solutions containing DEAE provides a slightly better protection than FFA solution with $\mathrm{CHA}$. Thus, the more toxic CHA can be replaced by DEAE.

Keywords: film formation, electrochemical impedance spectroscopy, oleyldiamine, cyclohexylamine, diethylaminoethanol, carbon steel.

Received: September 11, 2015. Published: October 21, 2015.

doi: $\underline{10.17675 / 2305-6894-2015-4-4-5}$ 


\section{Introduction}

Film forming amines (FFAs), also called fatty amines or polyamines, are used as corrosion inhibitors in closed cooling/heating systems [1] as well as in steam generators [2-5]. Due to their volatility, they are able to protect the whole water/steam cycle by the formation of a thin film on the metal surfaces, which acts as a barrier between the corrosive agents in the water and the metal.

In steam generators film forming amines are generally applied in combination with alkalising amines (AAs) e.g. cyclohexylamine (CHA) or diethylaminoethanol (DEAE), which adjust the $\mathrm{pH}$ to an alkaline range and minimise the solubility of iron. DEAE is proposed as replacement for CHA, which is suspected of damaging fertility.

Although the film formation by FFA has been subject of numerous studies [6-10], much less is known on the influence of AA on the film formation. There is a concern, whether the change about the alkalising amine due to their different hydrotropy might impact the protective properties of filming amines and thus might lead to corrosion.

Therefore, the film formation of oleyldiamine, a commonly used FFA, on carbon steel has been studied with electrochemical impedance spectroscopy (EIS) in defined test water with and without the presence of different AAs. The studies have been carried out at two different $\mathrm{pH}$ values over an exposure time up to $5 \mathrm{~h}$.

\section{Experimental}

Electrochemical impedance spectroscopy was used to characterize the inhibitive properties of three different inhibitor solutions based on FFA and to compare their anticorrosion efficiency with untreated medium (blank).

\subsection{Test solutions}

Three different corrosion inhibitors were studied in the form of aqueous solutions for corrosion protection of water/steam cycles. The inhibitor compositions are given in Table 1. In water/steam cycles DI-water is generally used with a very low electrical conductivity $(<1 \mu \mathrm{S} / \mathrm{cm})$. In order to ensure the necessary electrical conductivity needed for the measurement $200 \mathrm{mg} / \mathrm{L} \mathrm{NaCl}$ were added.

The concentration of FFA was chosen to provide enough material to establish a complete film on the electrode. The AA concentrations were chosen to achieve the same $\mathrm{pH}$-value in the solution, taking into account the different basicity of the two molecules.

\subsection{Corrosion testing}

Electrochemistry is a powerful tool to study corrosion phenomena and widely applied in academic and industrial research [11-14]. Therefore, this method was chosen for this study.

Electrochemical measurements were carried out using an Autolab Metrohm frequency response analyser with an electrochemical interface. The impedance diagrams were plotted 
Table 1. Compositions of inhibitor solutions in DI-water. All solutions additionally contained $200 \mathrm{mg} / \mathrm{L}$ $\mathrm{NaCl}$.

\begin{tabular}{cccc}
\hline Inhibitor & $\begin{array}{c}\text { Oleyldiamine } \\
\text { FFA [mg/L] }\end{array}$ & $\begin{array}{c}\text { Cyclohexylamine } \\
\text { CHA [mg/L] }\end{array}$ & $\begin{array}{c}\text { Diethylaminoethanol } \\
\text { DEAE [mg/L] }\end{array}$ \\
\hline Blank & 0 & 0 & 0 \\
FFA & 4 & 0 & 0 \\
FFA/CHA & 4 & 14 & 0 \\
FFA/DEAE & 4 & 0 & 22 \\
\hline
\end{tabular}

at the corrosion potential after 2 hours of immersion in a frequency range of $65 \mathrm{kHz}$ to a few $\mathrm{mHz}$ with eight points per decade. The electrochemical results were obtained from a minimum of five subsequent experiments. For the study of the film formation in time a maximum of 15 measurements were carried out with 20 minutes delay between the start of each one.

The corrosive medium was a $200 \mathrm{mg} / \mathrm{L} \mathrm{NaCl}$ solution in demineralized water in contact with air maintained at $25^{\circ} \mathrm{C}$. The choice of this medium was based upon the following criteria [6]: $(i)$ its low electrical conductivity is close to that encountered in industrial water circuit, (ii) its corrosivity is fairly high, and (iii) it is an easily reproducible baseline solution.

Depending on the concentration of the compounds, the $\mathrm{pH}$ of the inhibitive solutions varied between 7 and 10. The $\mathrm{pH}$ was adjusted to the desired value by adding $\mathrm{NaOH}$ (for $\mathrm{pH}$ increasing) or $\mathrm{HCl}$ (for $\mathrm{pH}$ decreasing).

Representing the most important material used in the water/steam cycles carbon steel was used as sample material. For all the experiments, the sample materials were polished in three steps with $\mathrm{SiC}$ paper from grade 1200 to grade 4000, cleaned in ethanol and demineralized water and then dried in warm air.

For the test samples, the working electrode was a rotating disc consisting of a rod of $1 \mathrm{~cm}^{2}$ cross-sectional area to ensure uniform thickness of the diffusion layer at the electrode surface and a heat-shrinkable sheath leaving only the tip of the cylinder in contact with the solution. All experiments were carried out at a rotation speed of $500 \mathrm{rpm}$. A saturated calomel electrode (SCE) was used as reference and the counter-electrode was a platinum grid.

The carbon steel/solution interface was mathematically described in terms of an electric equivalent circuit consisting of a resistance electrolyte in series with one or two parallel constant phase element (CPE)/resistor combinations. The semicircular shape of the impedance diagrams allowed the modeling with an equivalent circuit having the following characteristic parameters: $R_{\mathrm{p}}$, polarization resistance and CPE, double layer capacitance. The CPE which is described by two parameters (capacity $Y_{0}$ and exponent $n$ ) is generally attributed to the roughness and inhomogeneity of the surface or to a non-uniform distribution of the current density on the electrode undergoing corrosion $[15,16]$. 


\section{Results and discussion}

The EIS spectra obtained were fitted with the Autolab FRA software applying three different electric equivalent circuits EEC (Figure 1). Although single EIS spectra could probably have been described better by different EEC, it was decided to always apply the same model within spectra series with different exposure times.

The spectra of blank solution could be described well with the simple EEC $_{a}$ (Figure 1a). The spectra of solutions containing FFA could be described only with more complex EEC (Figure 1b, c). Since the data obtained by the fits for the EECs with two CPE did not differ significantly (Table 2), in this paper only the data of the fit with $\mathrm{EEC}_{\mathrm{b}}$ are shown. For the graphical presentation of the spectra the Nyquist plot has been chosen. The comparison of measured and fitted data is shown for two examples in Figure 2.

$\mathrm{EEC}_{\mathrm{a}} \quad \mathrm{EEC}_{\mathrm{b}} \quad \mathrm{EEC}_{\mathrm{c}}$

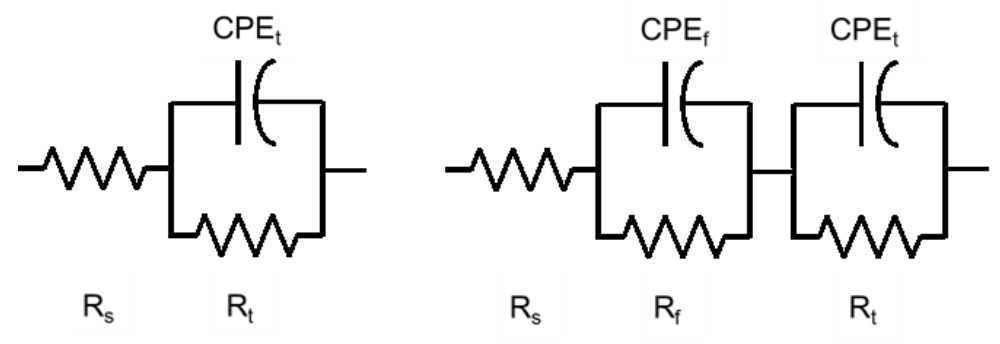

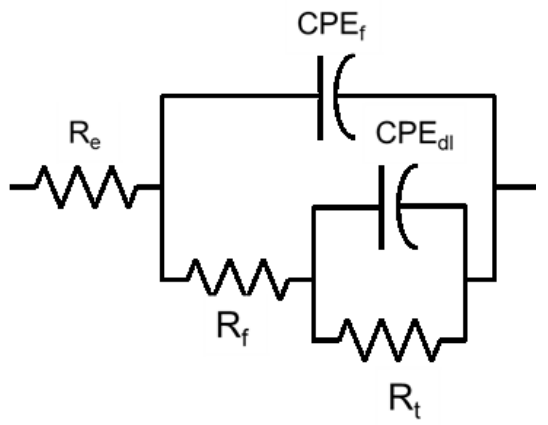

Figure 1. Electrical equivalent circuits (EEC) used for the mathematical description of the Electrochemical Impedance Spectra.

Table 2. Comparison of fitting data obtained for two different electrical equivalent circuits. The example shows the data for FFA/DEAE at $\mathrm{pH} 8.2$ after 20 minutes exposure time.

\begin{tabular}{cccc}
\hline Parameter & Unit & $\mathbf{E E C}_{\mathbf{b}}$ & $\mathbf{E E C}_{\mathbf{c}}$ \\
\hline$R_{\mathrm{s}}$ & {$\left[\mathrm{k} \Omega \cdot \mathrm{cm}^{2}\right]$} & 0.205 & 0.206 \\
\hline $\mathrm{CPE}_{\mathrm{f}}$ & & & \\
$Y_{0}$ & {$\left[\mathrm{~F} / \mathrm{cm}^{2}\right]$} & $1.4 \mathrm{E}-7$ & $1.1 \mathrm{E}-7$ \\
$n$ & & 0.808 & 0.802 \\
\hline$R_{\mathrm{f}}$ & {$\left[\mathrm{k} \Omega \cdot \mathrm{cm}^{2}\right]$} & 0.707 & 0.813 \\
\hline $\mathrm{CPE}_{\mathrm{t}}$ & & & \\
$Y_{0}$ & {$\left[\mathrm{~F} / \mathrm{cm}^{2}\right]$} & $2,4 \mathrm{E}-7$ & $3.5 \mathrm{E}-7$ \\
$n$ & & 0.614 & 0.625 \\
\hline$R_{\mathrm{t}}$ & {$\left[\mathrm{k} \Omega \cdot \mathrm{cm}^{2}\right]$} & 2.31 & 2,20 \\
\hline Rel. Chisqr & & $1.86 \mathrm{E}-2$ & $1.84 \mathrm{E}-2$ \\
\hline
\end{tabular}



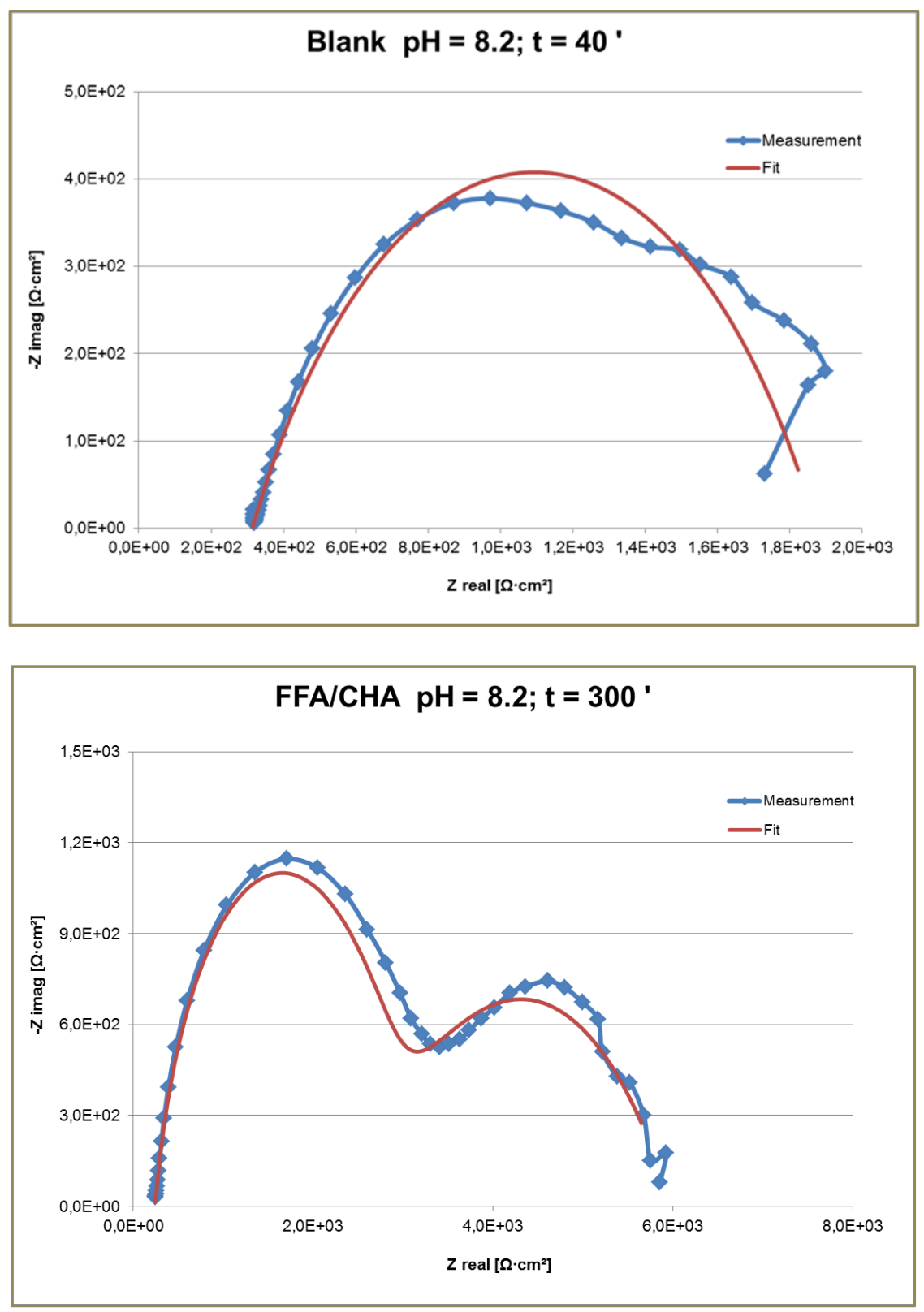

Figure 2. Comparison of measured and fitted data of Electrochemical Impendence Spectra for: top: Blank fitted with electrical equivalent circuit $a\left(\mathrm{EEC}_{\mathrm{a}}\right)$; bottom: $\mathrm{FFA} / \mathrm{CHA}$ fitted with electrical equivalent circuit $b\left(\mathrm{EEC}_{\mathrm{b}}\right)$.

\subsection{Spectra dependence on inhibitor solution}

The four test solutions show very different EIS spectra: The spectra of the blank is characterised by one asymmetrical semi-circle, whereas the spectra of the solutions containing film forming amine show the typical two semi-circles. In the spectrum of the solution containing only film forming amine the two semi-circles cannot be easily distinguished. Fitting with only one $\mathrm{CPE}\left(\mathrm{EEC}_{\mathrm{a}}\right)$, however, did not provide a satisfying result. Figure 3 shows the Nyquist plots of the EIS spectra for the four solutions at a $\mathrm{pH}$ of 8.2 after 300 minutes of exposure time. 


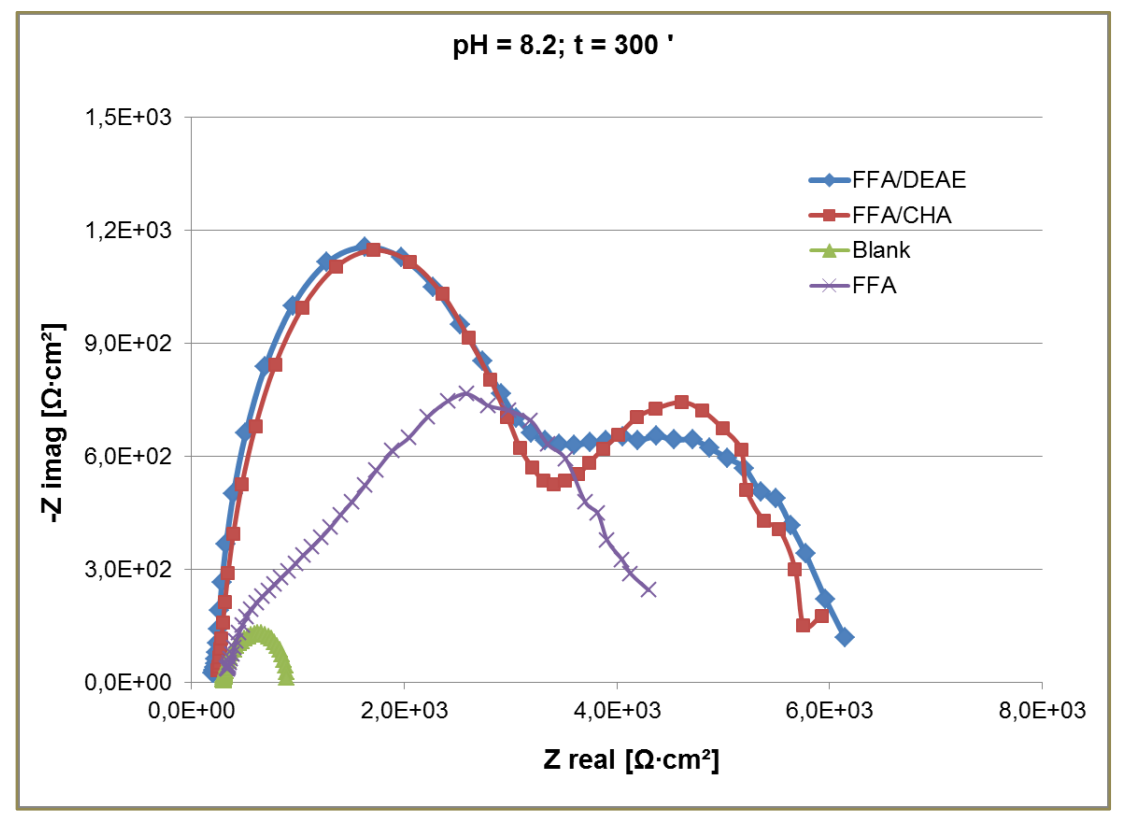

Figure 3. Comparison of Electrochemical Impendence Spectra for three different inhibitor solutions based upon film forming amine (FFA) and solution without inhibitor (Blank).

It is clearly visible that the polarisation resistance for the three solutions with inhibitor based on FFA is roughly one order of magnitude higher than the solution without inhibitor. Both solutions containing additionally alkalising amines (AA) have a higher polarisation resistance than the solutions containing only FFA. There is no significant difference between the results of the two FFA + AA solutions.

\subsection{Spectra dependence on time}

For the solutions at $\mathrm{pH} 8.2$ a series of 15 measurements over a period of totally 300 minutes have accumulated for each inhibitor solution. It can be noted that the shapes of the EIS curves significantly change during the total measuring time of 300 minutes. Figure 4 shows as an example five EIS spectra of the series for the blank and the FFA solution with CHA.

There is marked difference between the time dependence of the two EIS spectra shown: the polarisation resistance decreases with time for the blank solution (top), whereas it strongly increases for the FFA solution with CHA (bottom). The high frequency semicircle increases in diameter, the low frequency semi-circle is more or less constant. Apparently, the film formation process has not come to equilibrium at the end of the total exposure time. The same is valid for the FFA/DEAE solution. 

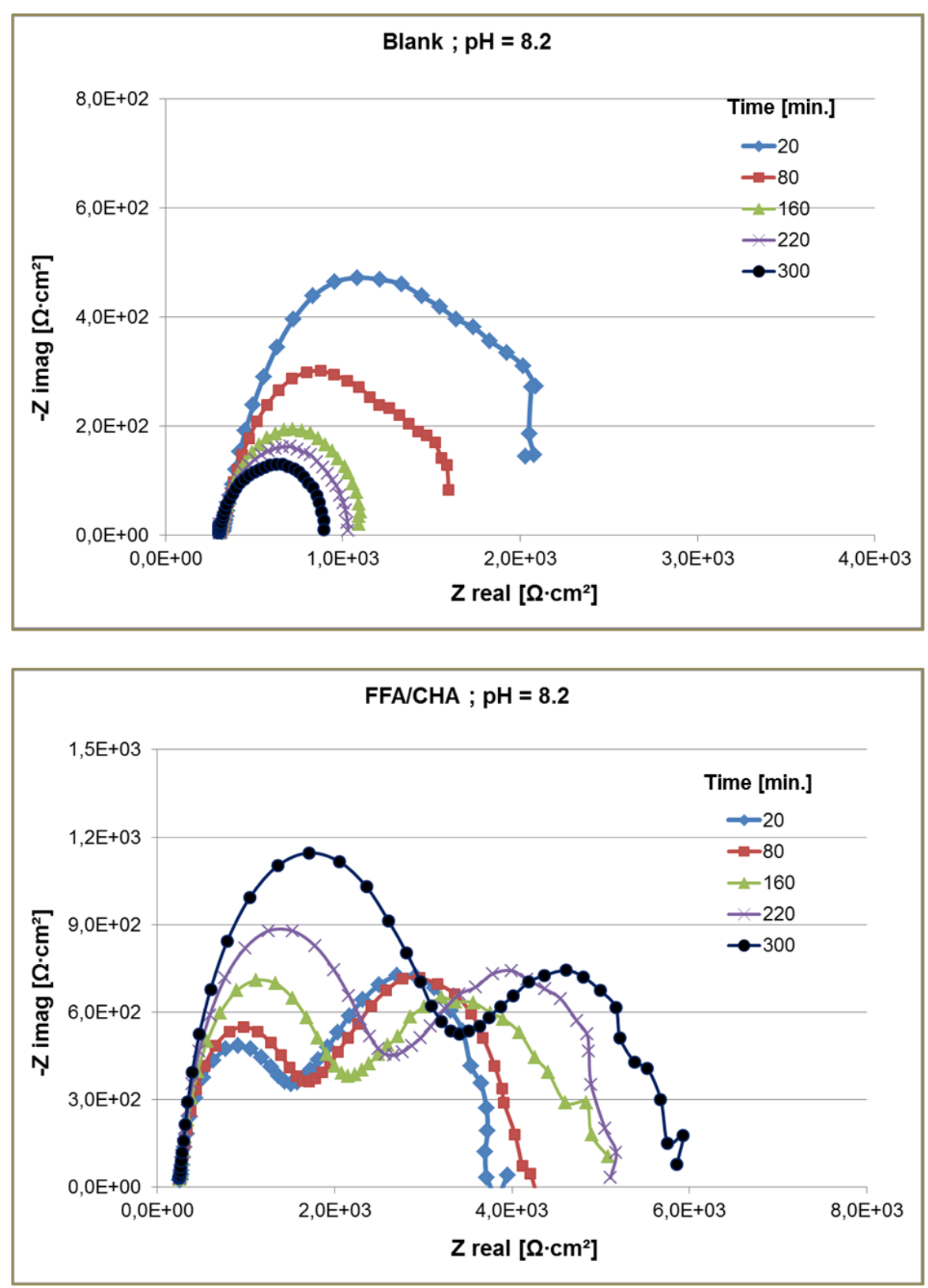

Figure 4. Dependence of Electrochemical Impendence Spectra on time for the solution without inhibitor (Blank) (top) and the solution based upon film forming amine and cyclohexylamine (FFA/CHA) (bottom). Note that the scales of the axes are different.

For all four solutions, the solution resistance remains constant over time and is $0.30 \mathrm{k} \Omega \cdot \mathrm{cm}^{2}$ resp. $0.31 \mathrm{k} \Omega \cdot \mathrm{cm}^{2}$ for the solutions without AA and $0.20 \mathrm{k} \Omega \cdot \mathrm{cm}^{2}$ resp. $0.24 \mathrm{k} \Omega \cdot \mathrm{cm}^{2}$ for the solutions with AA. The lower resistance of the solutions containing AA reflects their higher electrical conductivity.

Figure 5 shows the development of the polarisation resistance $R_{\mathrm{P}}$ and the parameters of the CPE with time. For the film forming amine solutions, the data of the high frequency loop are presented. 
The polarisation resistances of the two AA containing solutions continuously increase and have more or less the same value, both reaching a value of approx. $6 \mathrm{k} \Omega \cdot \mathrm{cm}^{2}$ at the end of the experiment. The absolute differences should be critically viewed due to the scattering of data. The polarisation resistances of the solution containing only film forming amine are roughly constant $\left(4.2 \pm 0.4 \mathrm{k} \Omega \cdot \mathrm{cm}^{2}\right)$. In contrast to this the polarisation resistance of the blank decreases over time and finally reaches $0.6 \mathrm{k} \Omega \cdot \mathrm{cm}^{2}$. The decay roughly follows an exponential law. The corrosion protection achieved by the inhibitor solutions decreases in the order: FFA/DEAE $\approx$ FFA/CHA $>$ FFA $>$ Blank.

From the time dependence of the polarisation data the conclusion can be drawn that the layer formation has not come to equilibrium for the blank and the film forming amine solutions containing alkalising amines (FFA/DEAE and FFA/CHA). A closer look on the data of the constant phase elements proves that in spite of the constant polarisation resistance the layer formation is not finished for the solution containing only FFA, as both parameters (capacity $Y_{0}$ and the exponent $n$ ) significantly and systematically change over time (Figure $5 b, c$ ).

The continuous increase of $n$ from 0.26 at the beginning to 0.55 at the end of the experiment indicates an increasing homogeneity of the layer. Furthermore, the increase of $Y_{0}$ shows a change of its porosity. This could be interpreted as a reorganisation of the film.

The inhomogeneity of the layer constantly increases over time for the blank: The exponent decreases almost linear with time from 0.64 to 0.52 . The corrosion process of the metal creates a less and less protective layer.

The constantly high exponent $n$ between 0.82 and 0.96 of the solutions containing FFA and alkalising amines shows almost the behaviour of an ideal capacity. The value of the FFA/DEAE solution is a little higher. These two solutions form protective layers with low inhomogeneity within short term.

The marked difference in the layer formation between the two solutions containing FFA and AA and the solution containing only FFA can be plausibly explained by the hydrotropic effect of the AA: In the solutions without AA the FFA is probably existent at least partially as micelles. Bohnsack [17] states a critical micelle concentration (CMC) of oleyldiamine of 3 to $4 \mathrm{mg} / \mathrm{L}$, which is close to the concentration applied in this study.

It is evident that the film forming amine molecule shows a different adsorption and thus protection behaviour as a micelle or as an almost dissolved molecule. The concentration of FFA applied in practice is usually between 0.1 and $0.5 \mathrm{mg} / \mathrm{L}$, therefore, below the CMC. However, this concentration range would not allow the formation of a complete film on the vessel and electrode surfaces. For the objective of this study - to compare the effect of different alkalising amines on the film formation - this is of minor relevance. 

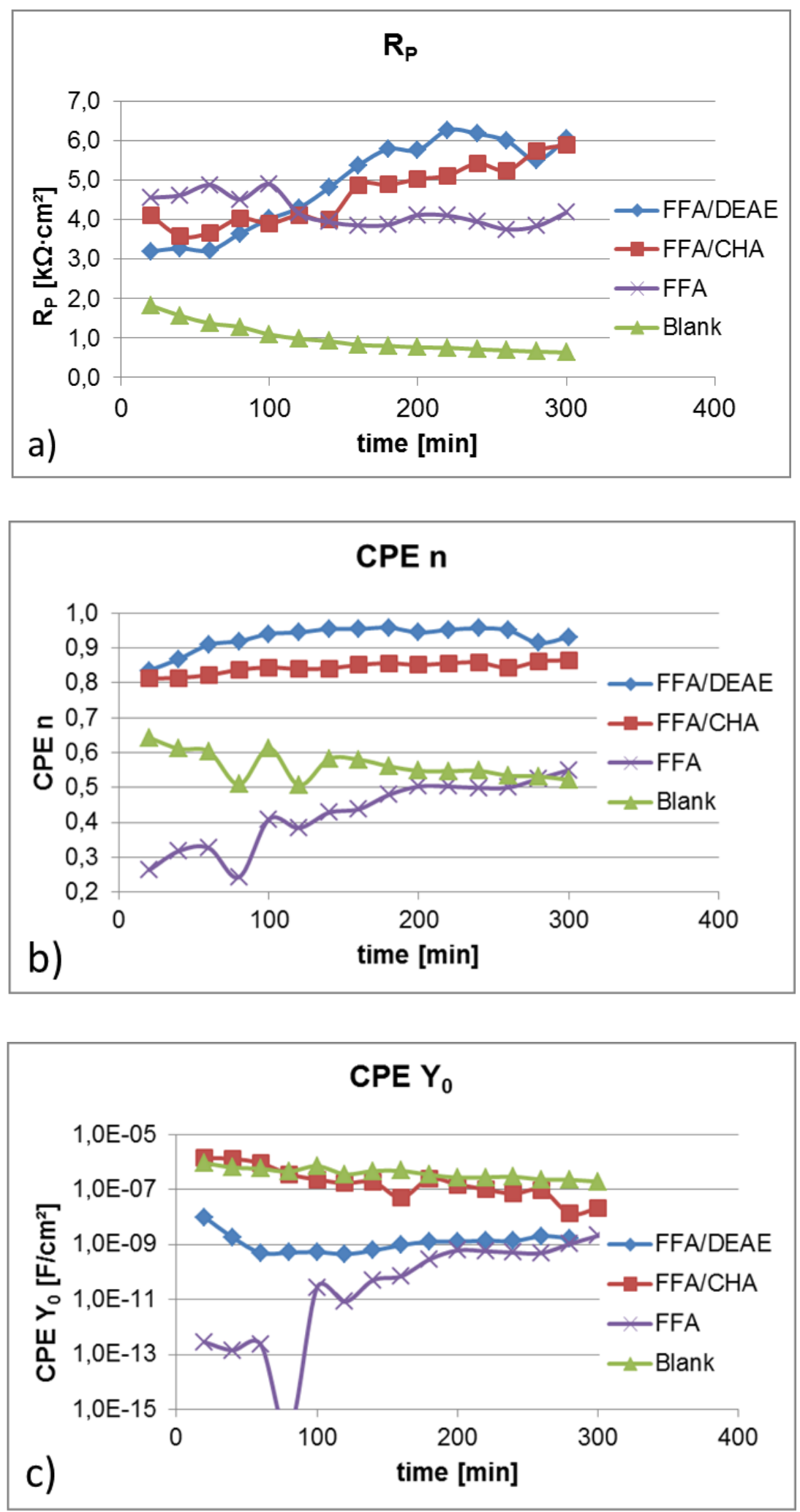

Figure 5. Dependence of $a$ ) polarisation resistance $R_{\mathrm{P}}$ and constant phase element (CPE) parameters; $b$ ) capacity $Y_{0}$ and $c$ ) exponent $n$ derived from Electrochemical Impedance Spectra on time for four different inhibitor solutions. For the solutions containing film forming amine (FFA) the CPE data of the high frequency semi-circle are shown. 


\section{3. pH dependence}

Additionally to the studies at $\mathrm{pH} 8.2$, which represent a situation of poor $\mathrm{pH}$ control in the water steam cycle, e.g. due to an ingress of a pollutant, EIS spectra were measured at $\mathrm{pH}$ 9.0, which is in the normal range of a well-controlled system. The EIS spectra were recorded over a period of 100 minutes. Unfortunately, the spectra of the inhibitor solution containing only film forming amine (FFA) showed high distortion. That is why the authors decided not to evaluate them.

The EIS spectra at $\mathrm{pH} 9.0$ for the two inhibitor solutions containing alkalising amines are dominated by the very large low frequency semi-circle (Figure 6). The EIS spectrum of the blank can only be seen in the detail. The polarisation resistances of the film forming amine solutions are almost two orders of magnitude higher than at a $\mathrm{pH}$ of 8.2 , whereas the value for the blank increases only slightly (Table 3 ).

The fitting data for the EIS spectra remain almost constant for the CPEs during the exposure time of 100 minutes. The polarisation resistances show the same dependence as for $\mathrm{pH}$ 8.2: increase over time for the FFA containing solutions $\left(117 \mathrm{k} \Omega \cdot \mathrm{cm}^{2}\right.$ to $263 \mathrm{k} \Omega \cdot \mathrm{cm}^{2}$ for FFA/DEAE, $92 \mathrm{k} \Omega \cdot \mathrm{cm}^{2}$ to $242 \mathrm{k} \Omega \cdot \mathrm{cm}^{2}$ for FFA/CHA); decrease for the blank $\left(2.40 \mathrm{k} \Omega \cdot \mathrm{cm}^{2}\right.$ to $\left.1.65 \mathrm{k} \Omega \cdot \mathrm{cm}^{2}\right)$. Generally, the polarisation resistance measured for FFA/DEAE is a little bit higher than for FFA/CHA.

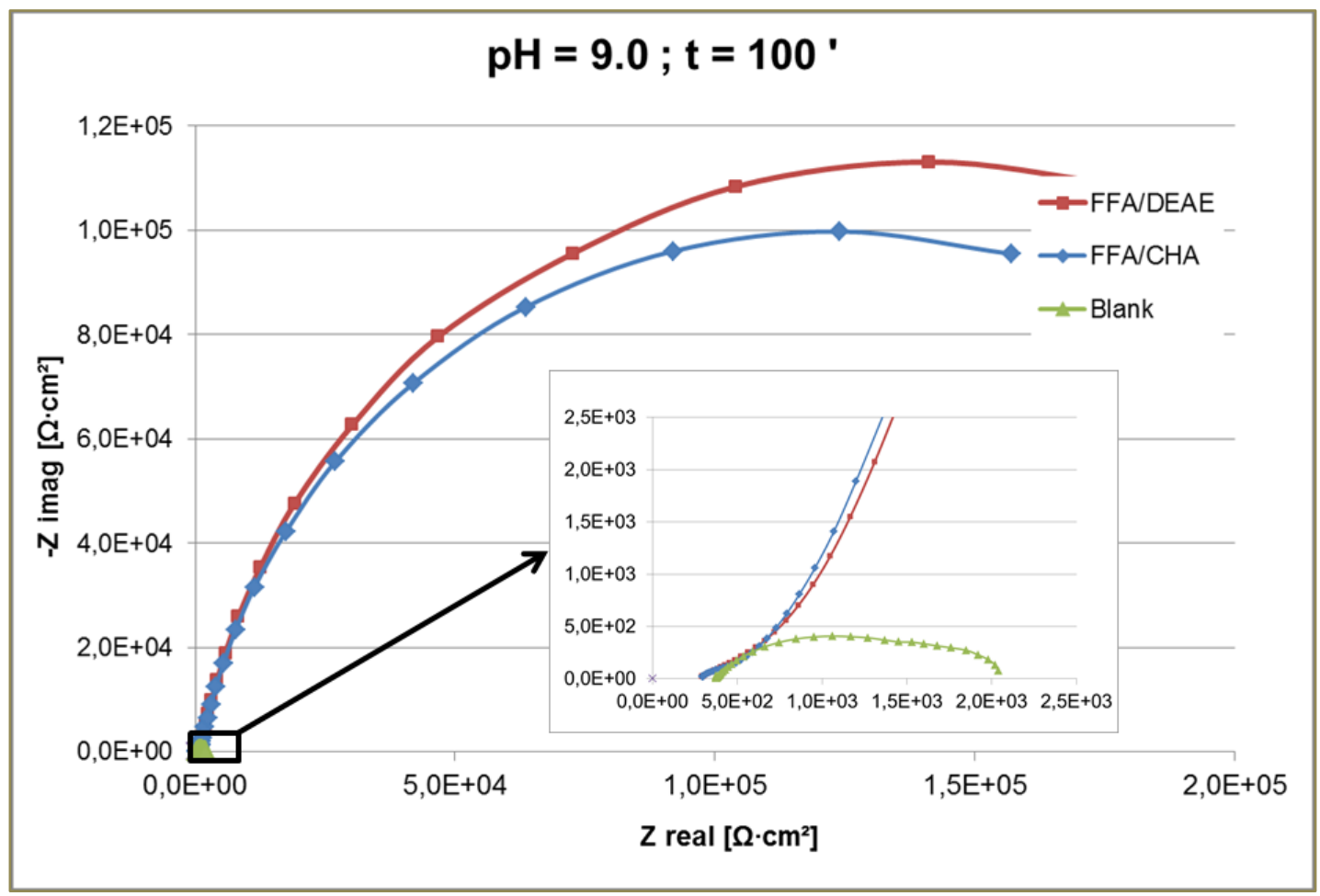

Figure 6. Comparison of Electrochemical Impendence Spectra for three different inhibitor solutions based upon film forming amine (FFA) and solution without inhibitor (Blank). 
Table 3. Comparison of polarization resistances at $\mathrm{pH} 8.2$ with 9.0 derived from electrochemical impedance spectra for four inhibitor solutions. The exposure time was 100 minutes.

Polarisation resistance

Inhibitor

$\left[\mathrm{k} \Omega \cdot \mathrm{cm}^{2}\right]$

\begin{tabular}{ccc}
\cline { 2 - 3 } & $\mathbf{p H}=\mathbf{8 . 2}$ & $\mathbf{p H}=\mathbf{9 . 0}$ \\
\hline FFA/DEAE & 4.01 & 264 \\
\hline FFA/CHA & 3.90 & 242 \\
\hline FFA & 4.90 & n.d. \\
\hline Blank & 1.09 & 1.65 \\
\hline
\end{tabular}

\section{Conclusions}

The corrosion protection of three inhibitor solutions based upon oleyldiamine, a frequently used film forming amine (FFA), alone and in combination with two alkalising amines has been studied by means of Electrochemical Impedance Spectroscopy (EIS) at two pH values in dependence on time. FFA based formulations are applied for the conditioning of water/steam cycle in power plant.

The main objective of this work was to investigate the impact of a substitution of cyclohexylamine (CHA), which is labelled H361f (suspected of damaging fertility) in FFA formulations by the non CMR labelled diethylaminoethanol (DEAE) on the film formation and thus on the corrosion protection.

All EIS spectra obtained for FFA containing solutions showed two semi-circles characteristic for the formation of a film on the surface. The film formation is not spontaneous and does not come to equilibrium state within the total exposure time of 300 minutes. At pH 9.0 the low-frequency semi-circle is predominant. The EIS spectra of the blank show a single asymmetric semi-circle.

All EIS spectra were mathematically described by mainly two different electric equivalent circuits using constant phase elements. The evolution of the parameters was determined over time. At both $\mathrm{pH}$ the polarisation resistance and thus the corrosion protection of the FFA and AA containing solutions increased significantly over time, whereas the blank showed a decrease. It remained almost constant for the solution only with FFA. The difference in behaviour between the FFA solution without and with AA is probably due to hydrotropic effect, which has a considerable influence under the conditions of the experimental set-up.

At $\mathrm{pH} 9.0$ the polarisation resistance was almost two orders of magnitude higher than at $\mathrm{pH}$ 8.2. The strongly improved corrosion protection cannot be explained by the different $\mathrm{pH}$, as the polarisation resistance of the blank only slightly increased.

According to the results of this study the cyclohexylamine, which is suspected to be toxic for reproduction can be replaced by the non CMR-labelled diethylaminoethanol 
without a deterioration of film formation and thus of the corrosion inhibition. The overall data may indicate even a slight improvement of the corrosion inhibition, when DEAE is used instead of CHA in combination with the film forming amine.

\section{References}

1. C. Forêt, G. Stoianovici, P. Bleriot, W. Hater and J. Matheis, PowerPlant Chem., 2014, 16, no. 6,361 .

2. W. Hater, C. Digiaro and C. Frayne, Int. Water Conf. 2012, San Antonio, Paper IWC $12-20$.

3. R. van Lier, G. Janssen and J. Savelkoul, VGB PowerTech., 2012, no. 8, 84.

4. B. Kolander, A. de Bache and W. Hater, PowerPlant Chem., 2013, 15, no. 2, 137.

5. W. Hater, A. de Bache and T. Petrick, PowerPlant Chem., 2014, 16, no. 5, 284.

6. M. Duprat, M. C. Lafont, F. Moran and F. Dabosi, Electrochim. Acta, 1985, 30, 353.

7. N. Ochoa, F. Moran and N. Pébère, J. Appl. Electrochem., 2004, 34, 487.

8. N. Ochoa, F. Moran, N. Pébère and B. Tribollet, Corros. Sci., 2005, 47, 593.

9. D. Frahne and T. Blum, PowerPlant Chem., 2006, 8, no. 1, 21.

10. C. Forêt, G. Stoianovici, G. Chaussec, A. de Bache, C. zum Kolk and W. Hater, Study of efficiency and stability of film forming amines (FFA) for the corrosion protection of the carbon steel in water circuits, EUROCORR 2008, Edinburgh, Paper no. 1404.

11. H. Wang, Electrochemical investigation of "green" film-forming corrosion inhibitors, Master Thesis, Division of Surface and Corrosion Science School of Chemical Science and Engineering KTH - Royal Institute of Technology Stockholm, Sweden 2011.

12. L. E. Tsygankova, V. I. Vigdorovich, N. V. Shel and E. V. Dubinskaya, Int. J. Corros. Scale Inhib., 2013, 2, 4, 304.

13. A. Frignani, C. Monticelli, F. Zucchi and G. Trabanelli, Int. J. Corros. Scale Inhib., 2014, 3, no. 2, 105.

14. S. Koehler and G. Reinhard, Int. J. Corros. Scale Inhib., 2014, 3, no. 4, 286.

15. C. Gabrielli, Identification of Electrochemical Processes by Frequency Response Analysis; Technical Report 004/83 of Solartron Analytical, Paris, 1980, 93.

16. G. J. Brug, A. L. G. Van Den Eeden, M. Sluyters-Rehbach and J. H. Sluyters, J. Electroanal. Chem., 1984, 176, 275.

17. G. Bohnsack, VGB KraftwerksTechnik, 1997, 77, no. 10, 841. 